\title{
La recesión y la medición de la tasa de desempleo de México ante una eventual reforma laboral
}

\begin{abstract}
Roberto Gutiérrez Rodríguez*
RESUMEN

En la antepropuesta de reforma a la Ley Federal del Trabajo, presentada por el Ejecutivo al Congreso en febrero de 2009, se muestran las incongruencias internas y externas de las estadísticas laborales de México y se analiza la conveniencia de aprovechar la coyuntura para que los representantes de los sectores productivos revisen su metodología de levantamiento, su consistencia histórica y su utilidad para tomar decisiones de política laboral. Se presta particular atención a la tasa de desempleo, cuyo nivel absoluto y variación a lo largo del tiempo difieren mucho de lo observado por los principales socios comerciales del país, agrupados en la Organización para la Cooperación y el Desarrollo Económicos (OCDE), con gran experiencia en materia de estandarización y procesamiento de este tipo de estadísticas. También se encuentran diferencias considerables con los de América Latina y el Caribe, a pesar de las similitudes culturales y de desarrollo, y aun mayores con los miembros del Tratado de Libre Comercio de América del Norte (TLCAN), particularmente Estados Unidos. En contraparte, el análisis sugiere un distanciamiento discreto respecto de la metodología de la Organización Internacional del Trabajo (OIT), cuya flexibilidad conduce a falta de precisión y puede afectar la imagen de una economía como la mexicana, que tiene muchos años luchando por situarse entre las primeras emergentes del mundo, pero que además de las fallas estadísticas descritas no cuenta con un seguro de desempleo, mientras los otros 30 miembros de la OCDE, incluyendo Chile, último país en incorporarse, tienen uno.
\end{abstract}

Palabras clave: reforma laboral, estadísticas laborales y tasa de desempleo.

Clasificación JEL: J21.

\begin{abstract}
The paper explores the external and internal inconsistencies of Mexico's labor force statistics and discusses the convenience of taking the February 2009 presidential proposition of reformulating the Federal Labor Law as an opportunity to encourage representatives of the productive sectors to review the underlying survey methodology as well as the historic consistency of labor force statistics and its appropriateness for policy-making. In this regard, particular attention is paid to the unemployment rate, whose absolute level and variations over time differ greatly from those observed by major Mexico's trading partners, grouped in the Organization for Economic Cooperation and Development (OECD), whose statistic division and working groups have a great deal of experience in the standardization and processing of this kind of statistics. There are also considerable differences with Latin America and the Caribbean countries, despite the similarities in culture and development, and even greater with the members of the North American Free Trade Agreement (NAFTA), particularly the USA. By contrast, the analysis suggests putting a discreet distance from the International Labor Organization's methodology (ILO), whose flexibility leads to lack of precision and can affect the image of an emerging economy struggling to become one of the first of its class. It is convenient to bear in mind that in addition to labor force statistics weaknesses Mexico has not an unemployment insurance, while the other 30 OECD members, including Chile, the last country joining the organization (January 2010) have one in place.
\end{abstract}

Key words: Labor reform, labor statistics, unemployment rate.

Classification JEL: J21.

* Profesor-investigador de la Universidad Autónoma Metropolitana, Xochimilco. Agradezco a dos dictaminadores anónimos sus acertadas observaciones, sin que ello los comprometa con los argumentos centrales ni con las fallas finales, que son de mi exclusiva responsabilidad. 


\section{INTRODUCCIÓN}

El 9 de febrero de 2009 el secretario del Trabajo y Previsión Social (STPS) de México presentó al Poder Legislativo un documento basado en presuntas propuestas elaboradas por diferentes partidos e instituciones, y recogidas por el Ejecutivo (Lozano, 2009), cuyo fin sería motivar una reforma a la Ley Federal del Trabajo (LFT). ${ }^{1}$ La antepropuesta, como se le ha llamado porque su formalización se llevaría a cabo en 2010, parte de un principio concreto, aunque subrepticiamente manejado: que los trabajadores acepten una serie de obligaciones vinculadas con la elevación de la productividad y la competitividad de las empresas, al tiempo que renuncian a ciertos derechos presentes en la LFT reformada por última vez en 1980, y en los contratos colectivos de trabajo (CCT). Todo lo anterior, a cambio de una expectativa de mejoramiento de largo plazo de sus remuneraciones y de mejores oportunidades de empleo y condiciones de trabajo para el país, junto con una eventual reducción de la informalidad.

El problema en que se centra este trabajo, y que se desprende de la antepropuesta presentada por la STPS, es que para satisfacción de los trabajadores y un mejor diseño de la futura política laboral de México, los representantes de los empresarios y el gobierno deberían acceder a revisar la metodología de levantamiento de las estadísticas laborales del país, sobre todo la tasa de desempleo. Esto en virtud de que: $a$ ) no se ajustan al nuevo marco internacional de México; $b$ ) están diseñadas para ofrecer una visión optimista del mercado laboral; c) padecen de problemas de discontinuidad, y $d$ ) al no reconocer el grave deterioro del mercado de trabajo tampoco aceptan la necesidad de su solución.

En este contexto, el objetivo del documento es analizar la metodología de captación y medición de las estadísticas laborales del país a partir de la oportunidad de diálogo que ofrece la eventual reforma a la LFT. En tanto, la hipótesis es la siguiente: en la medida en que estén mal cuantificadas las estadísticas laborales, sobre todo la tasa de desempleo, seguirá siendo equivocado el diseño de la política laboral, en detrimento de los trabajadores formales e informales, así como de algunos frentes de la seguridad social de México.

${ }^{1}$ En la LIX Legislatura (2003-2006) se presentaron 157 propuestas de reforma a la normatividad laboral: 88 sobre la LFT, 54 a otras disposiciones en la materia y 15 dirigidas a la codificación laboral y otros aspectos. En la LVII Legislatura se habían presentado 37 propuestas y en la LVIII Legislatura 53. Con esto, entre el $1^{\circ}$. de septiembre de 1997 y el 21 de agosto de 2006 se acumularon 247 iniciativas (CESOP, 2009). 
El contenido del documento es como sigue: en la sección I se analiza la legislación del país en el campo de la estadística, particularmente la referida al poder casi omnímodo del Instituto Nacional de Estadística y Geografía (INEGI) en la materia. En la sección II se reflexiona sobre los problemas de medición de las estadísticas laborales, principalmente la tasa de desocupación/desempleo, elaborada por dicho instituto. En la sección III se analiza la consistencia interna de las cifras de ocupación de la ENOE, a partir de la evolución reciente de las variables PEA, PNEA y población en edad de trabajar. En la sección IV se pone en evidencia la pertinencia de las estadísticas laborales de México por medio de algunas comparaciones con el resto de países miembros de la Organización para la Cooperación y el Desarrollo Económico (OCDE), el Tratado de Libre Comercio de América del Norte (TLCAN) y la región de América Latina y el Caribe. Al final, se presentan las conclusiones.

\section{LA LEGISLACIÓN EN MATERIA ESTADÍSTICA}

Las dos principales propuestas de reforma a la LFT, o por lo menos las que más se han difundido - la tripartidista del Partido Acción Nacional (PAN), el Partido Revolucionario Institucional (PRI) y el Partido Verde Ecologista de México (PVEM), también conocida como Ley Abascal presentada en 2002, así como la actual (Lozano, 2009)-, omiten los obvios problemas de levantamiento de las estadísticas laborales de México y de medición de la tasa de desempleo.

El hecho de que la nueva Ley del Sistema Nacional de Información Estadística y Geográfica (Diario Oficial de la Federación, 2008) acordada por el Poder Legislativo con el fin de transformar al entonces Instituto Nacional de Estadística, Geografía e Informática en Instituto Nacional de Estadística y Geografía (en ambos casos INEGI) y así lograr que el diseño, levantamiento y presentación de las estadísticas de México se pusiera al servicio de la sociedad y en él pudieran verse reflejados los intereses y necesidades de todos sus miembros (lo cual no se logró), muestra una vez más cómo en nuestro país las cosas cambian para seguir igual.

Es decir, por un largo periodo - por lo menos desde que Vicente Fox llevó a cabo su campaña presidencial en 2000, hasta pasado el segundo año de la administración de Felipe Calderón - se discutió la conveniencia de ciudadanizar el INEGI, pues sólo de esa manera se justificaría transformarlo de órgano desconcentrado de la Secretaría de Hacienda y Crédito Público (SHCP) en institución 
autónoma al servicio de la sociedad. Al final, la ley modificada tuvo otros resultados. Por una parte, dispone que el INEGI es "un organismo público con autonomía técnica y de gestión, personalidad jurídica y patrimonio propios" (artículo 52). Por otra, acota que el Consejo Consultivo del Sistema Nacional de Información Estadística y Geográfica estará constituido por funcionarios de los poderes Ejecutivo, Legislativo y Judicial, así como cinco representantes de las entidades federativas más uno del Banco de México (artículo 14).

Con la modificación anterior, el INEGI deja atrás su dependencia administrativa de la SHCP y asume el control del levantamiento de los índices de precios al productor y al consumidor (inflación); pero no por ello se ciudadaniza ni da oportunidad de participar a la sociedad civil -investigadores, estadígrafos y eventualmente expertos internacionales- en el diseño de las encuestas y en el contenido, levantamiento y difusión de las estadísticas del país. Todo lo contrario, como consta en el Diario de Debates de la Cámara de Diputados de principios de abril de 2008, con la nueva ley el Ejecutivo por lo menos mantiene su poder en el instituto, ya que directamente nombra al presidente del INEGI, quien como trámite, debe ser ratificado por el Poder Legislativo.

Y si tanta fuerza tiene el Ejecutivo en el nombramiento del presidente del INEGI, su poder es al menos similar en cuanto al nombramiento de los miembros del Sistema Consultivo del Sistema Nacional de Información Estadística y Geográfica. Este método de trabajo, que el PRI desarrolló desde su fundación y puso en práctica durante 71 años, quedó a partir de 2000 al servicio de las iniciativas legislativas presentadas por el PAN. Así lo evidencia no sólo la reforma a la Ley del Sistema Nacional de Información Estadística y Geográfica, en marzo de 2008, sino la aprobación por parte de la Cámara de Senadores de los siete dictámenes de la reforma energética, en noviembre de 2008, entre los que se encontraban los de creación de la Comisión Nacional de Hidrocarburos, la Comisión Reguladora de Energía y la Ley de Petróleos Mexicanos, que considera el nombramiento de nuevos miembros en su Consejo de Administración (Pemex, 2009; Gutiérrez, 2009).

De lo anterior surgen problemas no sólo en la parte estadística, sino también en la informática: la decisión del Ejecutivo de arrancar al INEGI las funciones de su segunda "I", para transferirlas a un nuevo órgano, creado a principios de la administración Fox, e-México, condujo a resultados decepcionantes. Así lo corroboran dos hechos: en primer lugar, México redujo su posición en el ranking mundial de tecnologías de la información y la comunicación, al pasar del lugar 64 en 2002 al 75 en 2007 y, en segundo, lejos de convertirse en el primer país de 
la región de América Latina y el Caribe en materia informática, como se pretendía, ya en 2007 había sido desplazado al onceavo, ubicándose atrás de Argentina, Chile, Uruguay, Jamaica, Brasil, Panamá, Costa Rica, Venezuela, Colombia y Perú, en ese orden (ITU, 2009).

Como dato adicional, el artículo 9 de la Ley del Sistema Nacional de Información Estadística y Geográfica dispone la elaboración del Programa Estratégico del Sistema Nacional de Información Estadística y Geográfica, y del Programa Nacional de Estadística y Geografía. Empero, ninguno se encuentra disponible hasta el momento en los sitios oficiales (www.presidencia.gob.mx/ progsectoriales; www.inegi.org.mx; www.snieg.mx), a pesar de lo avanzado de la administración Calderón (la ley establece que estos documentos se deben publicar en el primer año de cada sexenio). Esto, aunado a la mala calidad de los planes y programas, junto con los pésimos resultados en materia de visualización de acontecimientos futuros, evidencia que la planeación y la programación se siguen contando entre las principales debilidades de las administraciones panistas.

\section{LA INADECUADA MEDICIÓN DE LA TASA DE DESOCUPACIÓN/ DESEMPLEO}

En este entorno de centralización estadística, que se remonta a antes de la creación del INEGI - inicios de la década de los ochenta- dicho instituto y la STPS decidieron, en 2004, hacer dos cambios aparentemente ascendentes, pero en realidad regresivos, a la información laboral obtenida vía encuestas.

En primer lugar, la Encuesta Nacional de Empleo (ENE) y su módulo urbano, la Encuesta Nacional de Empleo Urbano (ENEU), se transformaron en Encuesta Nacional de Ocupación y Empleo (ENOE), supuestamente con el fin de tomar en cuenta los compromisos y necesidades de homologación estadística de México con la OCDE y el TLCAN. Vale la pena ver cómo lo describe el propio INEGI:

La Encuesta Nacional de Ocupación y Empleo (ENOE) es la consolidación y fusión de la Encuesta Nacional de Empleo Urbano (ENEU) y la Encuesta Nacional de Empleo (ENE), que por más de 20 años estuvieron proporcionando información de la población ocupada y desocupada. A su vez, éstas son resultado de una larga tradición de encuestas en hogares que dio inicio en 1972 con el levantamiento de la Encuesta Nacional de Hogares (ENH) continuando en 1973; durante 
1973 y 1974 la Encuesta Continua de Mano de Obra (ECMO); de 1974 a 1984 la Encuesta Continua sobre Ocupación (ECSO); de 1983 a 2004 la Encuesta Nacional de Empleo Urbano (ENEU) y de 1991 a 2004 la ENE.

La ENOE es resultado de una evaluación integral de varios años, durante los cuales el INEGI ha realizado una serie de actividades de carácter conceptual, metodológico y de mejora de procesos, con la finalidad de presentar una nueva encuesta que permita captar y conocer de mejor manera las características del mercado laboral mexicano. Entre dichas actividades se encuentra la realización de talleres de consulta con usuarios tanto del sector académico como de instituciones públicas y un foro con personal de la OCDE; así como la ejecución de diferentes pruebas para los instrumentos de captación y los procesos de trabajo, entre ellas, una de significancia estadística con la idea de confrontar los resultados obtenidos con una análoga en dimensión y características extraída del levantamiento rutinario ENE-ENEU (INEGI, 2005a.)

En documentos posteriores, de carácter periódico, se hacen aclaraciones como la siguiente:

En el plano conceptual, la ENOE toma en cuenta los criterios que la OCDE propone dentro del marco general de la Organización Internacional del Trabajo (OIT), lo que permite delimitar con mayor claridad a la población ocupada y a la desocupada, además de facilitar la comparabilidad internacional de las cifras de ocupación y empleo. La ENOE, asimismo, incorpora el marco conceptual de la OIT y las recomendaciones del Grupo de Delhi relativas a la medición de la ocupación en el Sector Informal. La encuesta está diseñada para identificar sin confundir los conceptos de desocupación, subocupación e informalidad, así como también para tomar en cuenta y darles un lugar específico a aquellas otras personas que no presionan activamente en el mercado laboral porque ellas mismas consideran que ya no tienen oportunidad alguna de competir en él (mujeres que por dedicarse al hogar no han acumulado experiencia laboral, personas maduras y de la tercera edad, etcétera) (INEGI, 2009c.)

Empero, una revisión rápida de la metodología de la ENOE permite percatarse que los argumentos anteriores no son suficientes para evitar que el lector se vea orillado a tener, al menos, las cinco siguientes dudas: 
i) ¿Por qué la edad de trabajar, que se toma como base para calcular la tasa de participación en el trabajo y la PEA, es de 15 a 64 años en la OCDE en general, incluyendo Estados Unidos, y en México de 14 años y más, de modo que, independientemente del promedio de vida, la edad laborable para la OCDE es de 49 años en promedio y para México, con una expectativa de vida al nacer de 74 años, es de 60 ?

ii) ¿Por qué tomar como única referencia de la tasa de desocupación/desempleo abierto las encuestas en hogares, si los países más desarrollados de la OCDE, entre ellos Estados Unidos, tienen la posibilidad de confrontar las tendencias que arrojan éstas con el número de personas que solicitan el seguro de desempleo, lo que colateralmente implica contar con éste?

iii) ¿Por qué seguir tomando como mínimo una hora sin ocupación en la semana de referencia para considerar a la gente como desocupada, si para la elaboración de sus estadísticas de desempleo la OCDE parte en general de 15 horas laboradas y Estados Unidos en particular de 16?

iv) ¿Por qué no eliminar el concepto de trabajador no remunerado, con el que se encubre a millones de personas desempleadas, particularmente miembros de familias en las áreas rurales que se ocupan en labores casi improductivas al menos una hora a la semana, lo que en gran medida es inconsistente con la práctica estadística de la OCDE y de nuestros socios del TLCAN?

v) ¿Por qué seguir incorporando, a un ritmo tan acelerado, a los trabajadores desalentados al rubro de población no económicamente activa (PNEA)? Todos saben que éste es uno de los expedientes más recurrentes para reducir el renglón de desempleados, y que la oIT es demasiado flexible al respecto; así que si hasta cierto punto esto es aceptable, la flexibilidad internacional hace que algunos países rayen en el exceso.

vi) ¿Por qué cuantificar el subempleo o subocupación a partir del número de personas que, teniendo un trabajo, buscan conseguir un segundo, mientras en la OCDE en general el concepto se refiere a personas que trabajan menos de 30 horas a la semana? Curiosamente, las estadísticas del INEGI no levantan este dato. ${ }^{2}$

\footnotetext{
${ }^{2}$ Referencias estadísticas y metodológicas que se recomienda consultar sobre diferentes prácticas de clasificación de personas empleadas y desempleadas son: OIT (1986 y 1996), STPS (1994), Martin (1994) y BLS (2009).
} 
En segundo lugar, con la instauración de la ENEU se hicieron a un lado las razones por las que, progresivamente, el número de áreas urbanas que constituían la ENEU fue ascendiendo de 12 en 1985 a 48 a fines de los años noventa, y se decidió reducir la muestra tanto en número como en cobertura: pasó de 48 áreas urbanas a 32 ciudades, una por estado, como si éstas fuesen las únicas, o al menos las principales concentraciones de población representativas del empleo y desempleo citadino (ya no urbano) del país. Con la medida, se eliminaron áreas urbanas tan importantes para el monitoreo del empleo, el desempleo y otras variables laborales como Mexicali, Baja California; Ciudad Juárez, Chihuahua; Matamoros y Nuevo Laredo, Tamaulipas; Torreón y Monclova, Coahuila; Celaya y Salamanca, Guanajuato; Coatzacoalcos, Veracruz, y Tapachula, Chiapas.

Las decisiones tomadas por la STPS y el INEGI, además de la acotación geográfica antes señalada, condujeron a cuatro problemas:

i) A partir de 2005 se eliminaron diversas variables que levantaba la ENE, entre ellas las referidas al número total y distribución de la población asalariada: por sector de actividad, por tipo de remuneración, por tamaño de establecimiento, con y sin prestaciones, y con acceso a las instituciones de salud (Instituto Mexicano del Seguro Social [IMSS], Instituto de Seguridad y Servicios Sociales para los Trabajadores del Estado [ISSSTE], entre otras). También desaparecieron las tasas de ocupación de ambulantes y del sector no estructurado, algo sorprendente, dada la importancia que otorga la OIT a estos conceptos.

ii) Se inició el levantamiento de nuevas variables a partir del lanzamiento de la ENOE, particularmente: trabajadores subordinados y remunerados, así como su composición; población económicamente activa (PEA) disponible y no disponible; población subocupada y su composición, a partir del criterio de búsqueda de un segundo trabajo, no del número de horas trabajadas por semana; y nuevos tipo de unidades económicas: sociedades y corporaciones, negocios no constituidos en sociedad, privadas y públicas, informales, trabajo doméstico remunerado y agricultura de subsistencia.

iii) Las variables que se quedaron padecieron un quiebre a partir de 2005 debido a que la población en edad de trabajar, a partir de la que se calcu- 
lan múltiples variables del mercado laboral, entre ellas la tasa de participación en el mercado de trabajo, la tasa de ocupación y la tasa de desempleo, pasó de 12 a 14 años. Esta decisión se tomó, supuestamente, porque la LFT establece los 14 años como la edad en que, bajo ciertas condiciones -disponibilidad de certificado de primaria, permiso del padre o tutor, certificado médico y jornada diaria de no más de seis horas- el trabajo es legal. Empero, ni en eso nos unificamos con la OCDE, que toma como edad mínima los 15 años.

iv) La variable de población económicamente inactiva (PEI) se sustituyó por la de población no económicamente activa (PNEA). Aparentemente ambos términos son intercambiables, pero el INEGI no ha sido contundente al respecto.

Se deduce de los puntos anteriores que la ENOE se centra en el concepto de ocupación, el cual involucra el autoempleo y el trabajo familiar, mientras la ENE se centraba en el empleo, como hacen los países de la OCDE, incluyendo Canadá y Estados Unidos. Para no romper las series, el INEGI, con la anuencia de la STPS, ha llevado a cabo una labor de encadenamiento estadístico de la ENOE hasta 1995, montándose sobre las cifras de la ENE. Al hacerlo se han perdido las series de la ENEU; es decir, que las cifras de la muestra citadina (32 ciudades) no son compatibles, como era de esperarse, con la muestra urbana anterior (46 áreas urbanas). También se perdieron, evidentemente, las variables mencionadas en el inciso a). Las series que lograron conciliarse están disponibles en la página electrónica del INEGI a partir de 1995, aunque su difusión en medios impresos parte de 2005. Esto se puede verificar a través del Anuario estadístico de los Estados Unidos Mexicanos 2008 (INEGI, 2009a), que para todas las variables de la economía mexicana cubre el periodo 1980-2008, excepto para las laborales provenientes de encuestas, que apenas consideran el lapso 2005-2008.

Gráficamente, los dos hechos que más resaltan de la nueva serie son la brusquedad de los cambios entre 2003 y 2005, como muestra la gráfica 1, referida a la desocupación, y el acelerado crecimiento de dicha variable, tanto a nivel nacional como citadino (32 ciudades), durante los primeros siete meses de 2009, lo cual es consistente con las caídas del producto interno bruto (PIB) de $9.2 \%$ en el primer trimestre del año y de $10.3 \%$ en el segundo, ambas respecto a igual periodo del año anterior. 


\section{Gráfica 1. Tasas de desocupación nacional y urbana}

(Último trimestre de cada año, 2000-2009)

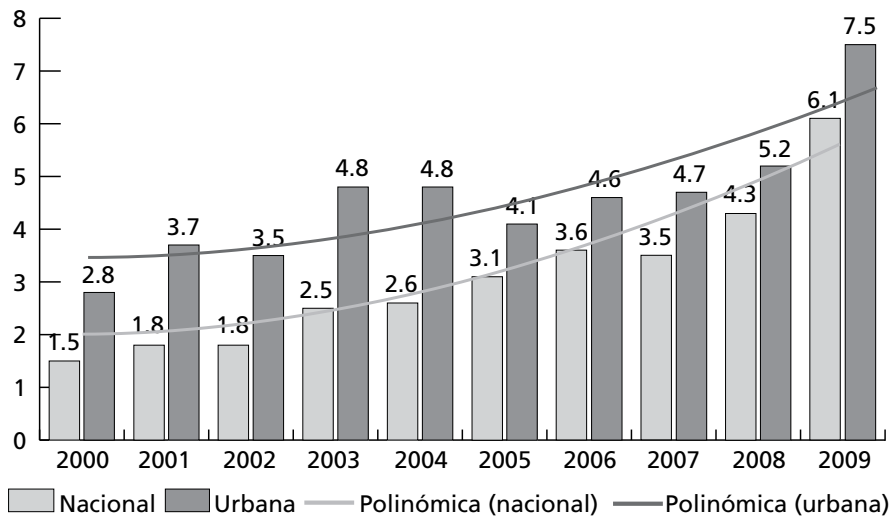

Fuente: INEGI/STPS (2009) e INEGI (2009d). Las cifras de 2009 corresponden a septiembre y han sido desestacionalizadas.

La gráfica también muestra que la tasa de desempleo nacional baja severamente en 2005 (instauración de la ENOE) respecto del año previo, en tanto que la urbana sube, y de ahí en adelante ambas aumentan a menor tasa, la primera, y mayor la segunda, por lo que sus líneas de tendencia se acercan a sólo nueve décimas una de la otra en 2008 (20\% de diferencia), no obstante que en 2003 la brecha era de 2.3 puntos porcentuales ( $92 \%$ de diferencia). Empero, en septiembre de 2009 las dos suben visiblemente, aunque la urbana lo hace más que la nacional y el diferencial se sitúa en 1.4 puntos porcentuales, o $23 \%$.

Los diferenciales anteriores muestran que dichas tasas no se pueden usar como sustitutas y que, cada vez que se hable de desocupación/desempleo en México, tendrá que aclararse si se trata de desocupación/desempleo nacional o urbano. Asimismo, se deberá hacer explícito si la tasa es normal o está desestacionalizada, como sucede con la correspondiente a septiembre de 2009, presente en la gráfica 1. Esta técnica permite suavizar el efecto de fenómenos coyunturales, como la incorporación de muchos jóvenes recién egresados de universidades e institutos al mercado laboral. Un ejemplo de estas diferencias es julio de 2009, cuando la cifra de desocupación nacional sin desestacionalizar fue de $6.12 \%$, mientras la desestacionalizada se ubicó en $5.7 \%$.

El impacto de las cifras sobre desempleo correspondiente a julio de 2009 , dadas a conocer por el INEGI el mes siguiente, fue tan grande para el propio gobierno federal o "gobierno del empleo" que, contra toda ortodoxia, la página electrónica de la STPS manifestó su inconformidad de la siguiente manera: 
La tasa de desocupación del mes de julio fue de $5.70 \%$, prácticamente la misma del pasado mes de junio (5.62\%), esto es, con una diferencia de 8 centésimas de punto porcentual. En este mismo sentido, es importante destacar que disminuyó la población subocupada ( $7.9 \%$ respecto de la población ocupada) al registrarse una baja de 1.13 puntos porcentuales respecto de junio de 2009. Lo anterior quiere decir que se registró un mayor número de personas que encontraron un trabajo de tiempo completo respecto del mes pasado (...) La tasa de desocupación más alta en la historia ha sido la del segundo trimestre de 1995 cuando alcanzó $6.86 \%$. Es muy importante destacar que este porcentaje se alcanzó por una gran pérdida de empleos, por efecto de la crisis económica interna. A diferencia de ello, la tasa de desocupación de $6.12 \%$ no se debe a una pérdida de empleos sino a que - además del contexto de crisis internacional de magnitud histórica en la que estamos inmersos - siempre en los meses de julio y agosto se registra presión adicional al mercado laboral de los estudiantes en el periodo vacacional. En otras palabras, un mayor número de personas que hasta hace poco eran inactivas, buscaron una ocupación. Lo que se comprueba con el resultado de la tasa neta de participación que se incrementó de $58.3 \%$ en junio a $59.0 \%$ en julio de 2009. Debe tenerse presente que ya en los meses de junio y julio, se observaron cifras positivas de trabajadores registrados ante el Instituto Mexicano del Seguro Social (STPS, 2009d).

Es evidente que la STPS, ya sea porque lo omite intencionalmente o porque lo desconoce, no menciona en su nota que:

a) La tasa de desocupación de $6.12 \%$ a que hace referencia sin justificación aparente es la misma de $5.70 \%$ que había mencionado antes, sólo que sin desestacionalizar.

b) Al revisar la tasa de desempleo urbano en el segundo trimestre de 1995 se observa que no fue de 6.86\%, sino de 6.5\% (INEGI, 1998).

c) No fue en el segundo trimestre de 1995, sino en el tercero, cuando se alcanzó la tasa de desempleo urbano más alta, por lo menos en la era de la ENEU, la cual llegó en agosto a 7.9\% (gráfica 2).

d) La tasa de desocupación de $5.7 \%$ correspondiente a julio de 2009 se refiere al ámbito nacional, mientras que la de $6.68 \%$ correspondiente al segundo trimestre de 1995 se refiere al ámbito urbano.

e) Para que se pueda comparar lo que sucedió en la recesión de 1995 con lo acontecido en la de 2009 es necesario partir de series homólogas, y éstas 
son las de desempleo/desocupación en el ámbito urbano, es decir la ENEU de 1995 (44 áreas urbanas en ese entonces) y la ENOE actual (32 ciudades). Los resultados se presentan en la gráfica 2, donde destaca que, mientras en julio de 1995 la tasa de desempleo urbano llegó a 7.3\%, en el mismo mes de 2009 la tasa de desocupación urbana desestacionalizada fue de $7.6 \%$, tres décimas de punto porcentual mayor, lo cual contradice el argumento que más preocupa a la STPS.

f) Gracias a que se dispone de cifras para septiembre de 2009 es posible observar que en dicho mes se iguala la tasa de desocupación urbana de la ENOE (7.6\%) con la de desempleo de la ENEU, correspondiente al mismo mes de 1995. La inflexión en la tendencia de la serie correspondiente a la recesión de 1995 fue posible gracias a que la economía respondió al estímulo de entonces a las exportaciones manufactureras, en virtud de la puesta en operación del TLCAN (enero de 2004). Desafortunadamente, dicho expediente se encuentra en un estado de maduración muy alto en 2010, por lo que es posible que los problemas de desocupación no cedan hasta entrado dicho año, como prevé la OCDE (OCDE, 2009a).

g) Aunque la tasa de participación en el trabajo aumentó ligeramente en el trimestre julio-septiembre de 2009, su nivel es muy inferior al alcanzado históricamente, lo que sugiere, como se argumentará más tarde, que no ha habido pulcritud en el manejo de las estadísticas laborales, problema sobre el que por supuesto no argumentó nada la STPS en su boletín de agosto (STPS, 2009b).

h) Las tendencias de la tasa de desocupación/desempleo del INEGI y de pérdida de plazas en el IMSS nunca han coincidido, por lo que no es correcto argumentar, con base en la generación de puestos de trabajo de carácter temporal, no permanentes, en empresas cuyos trabajadores cotizan para el instituto, que en el trimestre julio-septiembre se habría roto la tendencia a la baja del empleo. De hecho, lo que muestran las estadísticas es que en cada uno de estos meses el número de asegurados, tanto permanentes como eventuales, es considerablemente inferior al de igual mes del año anterior, y que las empresas siguen sin optar por contratar trabajadores de planta.

i) Es conveniente que la STPS lea cuidadosamente los reportes de empleo del INEGI, interprete adecuadamente los resultados de la ENOE y conozca cómo se vinculan con los del IMSS y de la economía en su conjunto. 
Gráfica 2. Tasas de desempleo urbano antes y durante dos recesiones: enero-agosto de 1993-1995, y enero-agosto de 2007-2009

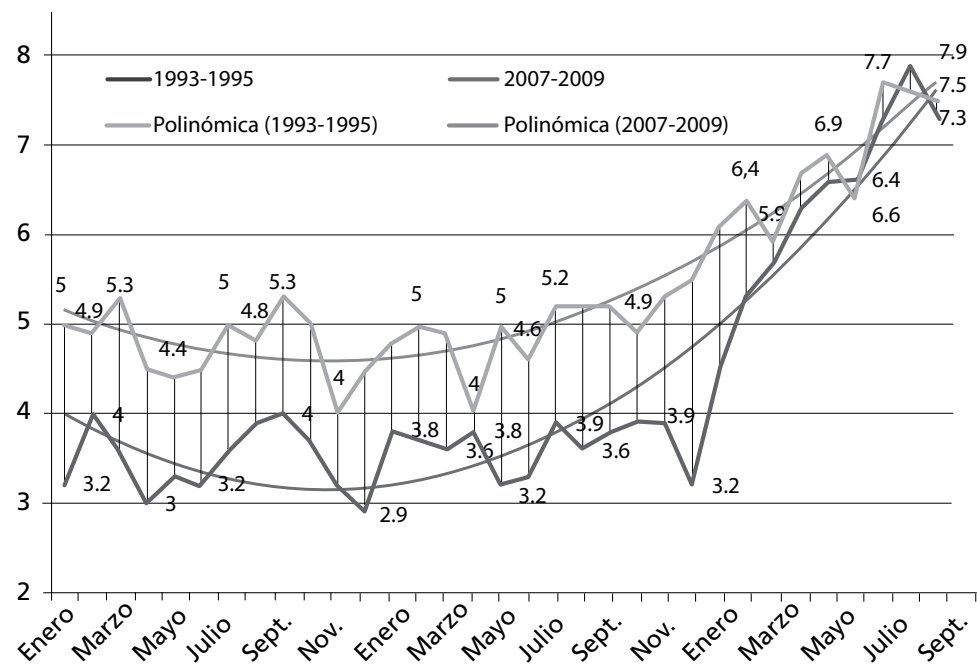

Fuentes: 1993-1995, INEGI (1998), a partir de ENEU, 44 áreas urbanas; 2007-2009, INEGI/STPS (2009) e INEGI (2009d), a partir de ENOE, 32 ciudades, cifras desestacionalizadas.

Por otra parte, al tratar de imponer como principal referencia de la evolución del mercado laboral de México la tasa de desocupación nacional en vez de la urbana - la que más usan los países que levantan encuestas laborales-, a diferencia de lo que sucedía en el pasado, la STPS y el INEGI muestran que su intención es tomar el indicador más conservador a su disposición. Esto en virtud de que, como es bien sabido, por razones metodológicas la tasa de desocupación en el campo es considerablemente menor a la urbana, y al promediar ambas la nacional resulta evidentemente menor a la urbana. Con esto queda claro, nuevamente, que el objetivo de las autoridades del país es presentar a México, a costa de lo que sea, como una de las naciones con la más baja tasa de desocupación del mundo. De ahí que, por eliminación, muchos analistas prefieran usar los registros administrativos del IMSS como indicadores de la evolución del mercado laboral.

Con la sustitución de la ENE por la ENOE se desaprovechó la oportunidad de corregir los errores de subestimación de los desempleados debido a la insistencia de que, con haber laborado al menos una hora en la semana de referencia - generalmente la previa al levantamiento de la encuesta - así sea como trabajador no remunerado (predominantemente de carácter familiar) o con tener una promesa de trabajo futuro, al encuestado no deberá considerársele desocupado. 
Y para llegar a dicha situación lo único que se requiere es que el encuestador del INEGI formule y enfatice sus preguntas en un orden previamente determinado por la institución (INEGI, 2005b).

\section{CONSISTENCIA DE LA TASA DE DESOCUPACIÓN CON OTRAS VARIABLES}

Una forma de ubicar las inconsistencias hasta ahora señaladas es a partir de la serie de tres cuadros que se presentan a continuación, referidos al incremento anual, en millones de personas, de distintas variables laborales.

En el cuadro 1 se muestra la población en edad de trabajar (14 años y más) que se incorporó al mercado entre enero de 2005 - año de inicio de la ENOE - y enero de 2009 (como se trata de incrementos, las cifras del primer año no aparecen). Los datos se separan entre PEA y PNEA. Debe observarse que la población en edad de trabajar acumulada en todo el periodo fue de 4.8 millones de personas, con un promedio anual de 1.2 millones; que de éstas, sólo 3.1 millones se transformaron en PEA, con un promedio anual de 775 mil, y que mientras entre enero de 2005 y el mismo mes de 2006 la PEA creció 1.1 millones, entre enero de 2008 y enero de 2009 apenas aumentó 100 mil.

\section{Cuadro 1. Composición de la población en edad de trabajar}

de enero de 2005 a enero 2009

(Incrementos anuales en millones)

\begin{tabular}{|c|c|c|c|}
\hline Año & Población en edad de trabajar & PEA & PNEA \\
\hline 2006 & 0.9 & 1.1 & -0.2 \\
\hline 2007 & 1.3 & 0.9 & 0.4 \\
\hline 2008 & 1.2 & 1.0 & 0.3 \\
\hline 2009 & 1.4 & 0.1 & 1.3 \\
\hline Acumulado & 4.8 & 3.1 & 1.8 \\
\hline
\end{tabular}

Fuente: a partir de STPS/INEGI (2009).

Lo anterior evidentemente es muy extraño, ya que si bien es conocida la aguda disminución de la tasa de natalidad de los últimos años, por razones de desfase su efecto en la PEA tomará varios años o lustros, como muestran Vela (2008) y otros demógrafos. De ahí que la bibliografía especializada se refiera, de manera insistente, al "bono demográfico", concepto que hace alusión al alto nú- 
mero de personas que se están incorporando al mercado laboral en la actualidad como resultado de las altas tasas de crecimiento demográfico del pasado y a las que, irónicamente, la economía no acierta a ofrecer empleos remuneradores.

Del consecuente incremento de la PEA, después de periodos con una alta tasa de crecimiento poblacional, dan cuenta las cifras del Consejo Nacional de Población (Conapo, 2009), cuya estimación para 2009 es un millón de personas superior a la de la ENOE. Evidentemente, las fricciones entre Conapo e INEGI por la veracidad de los cálculos han sido enormes; pero como por ley el INEGI es la autoridad estadística del país, las cifras del Conapo han tenido que circunscribirse a las proyecciones de las tasas de natalidad y mortalidad, así como de la población, lo mismo masculina que femenina. El resto de sus cálculos se deben tomar como información complementaria, sin carácter oficial.

En el cuadro 2 de la serie se muestra el incremento anual de la PNEA, separada entre PNEA no disponible (no buscaron trabajo en el periodo de referencia inmediato y no aceptarían uno aunque se les ofreciera) y PNEA disponible (no buscaron trabajo en el periodo de referencia inmediato, pero aceptarían uno al momento en que se les ofreciera). La cifra acumulada entre enero de 2005 y enero de 2009 de la PNEA no disponible es de 200 mil personas, mientras que la de la segunda es de 1.4 millones. En otras palabras, $78 \%$ de las personas que se incorporaron a la PNEA durante esos cuatro años manifestaron disposición para trabajar. Esto sugiere que la línea entre PNEA disponible y población desempleada es realmente tenue, por no decir arbitraria.

Cuadro 2. Composición de la población no económicamente activa (PNEA) (Incrementos anuales en millones)

\begin{tabular}{|c|c|c|c|}
\hline Año & PNEA & PNEA $^{*}$ & PNEA \\
\hline 2006 & -0.2 & -0.7 & 0.5 \\
\hline 2007 & 0.4 & -0.1 & 0.4 \\
\hline 2008 & 0.3 & 0.4 & -0.1 \\
\hline 2009 & 1.3 & 0.6 & 0.6 \\
\hline Acumulado & $\mathbf{1 . 8}$ & $\mathbf{0 . 2}$ & $\mathbf{1 . 4}$ \\
\hline
\end{tabular}

* No disponible.

Fuente: a partir de STPS/INEGI (2009).

En el cuadro 3 se hace un ejercicio, basado en las cifras de la ENOE, que pretende ejemplificar la forma en que el INEGI "acomodó" la PEA acumulada entre 
enero de 2005 y enero de 2009 (3.1 millones). Como se observa, la mayor parte (1.4 millones) se clasificó como PNEA disponible; le siguen los que lograron encontrar un empleo formal (apenas $900 \mathrm{mil}$ ), los que se fueron al desempleo abierto $(700 \mathrm{mil})$ y los que se ubicaron como trabajadores por cuenta propia (100 mil).

Cuadro 3. Acomodo de la PEA en principales componentes, enero 2005-2009 (Incrementos anuales en millones)

\begin{tabular}{|c|c|c|c|c|c|}
\hline Año & PEA & PNEA & Trab. formales & Desempleados & Por cta. prop. \\
\hline 2006 & 1.1 & 0.5 & 0.5 & -0.1 & 0.1 \\
\hline 2007 & 0.9 & 0.4 & 0.6 & 0.2 & -0.1 \\
\hline 2008 & 1.0 & -0.1 & 0.3 & 0.1 & 0.0 \\
\hline 2009 & 0.1 & 0.6 & -0.5 & 0.5 & 0.1 \\
\hline Total & 3.1 & $\mathbf{1 . 4}$ & $\mathbf{0 . 9}$ & $\mathbf{0 . 7}$ & $\mathbf{0 . 1}$ \\
\hline
\end{tabular}

Fuente: a partir de STPS/INEGI (2009).

Lo anterior se puede ver más claro en la gráfica 3, donde resalta que $45 \%$ de la nueva PEA se clasificó como PNEA disponible, a la que mayoritariamente la jerga laboral conoce como trabajadores desalentados, ya que supuestamente dejan de buscar empleo porque saben que no lo encontrarán. En seguida, aparecen los trabajadores formales, con 29\%; los desempleados, con 23\%, y los trabajadores por su cuenta, con $3 \%$.

Gráfica 3. Acomodo de la distribución de la nueva PEA entre enero de 2005 y enero de 2009. (Total: 3.1 millones de personas)

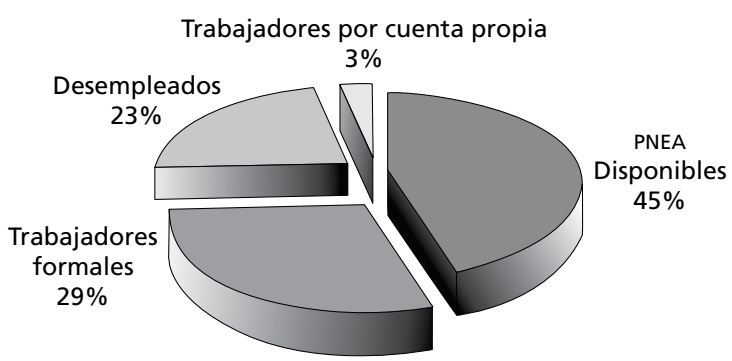

Fuente: con base en STPS/INEGI (2009).

En términos absolutos, el número total de personas contabilizadas por el INEGI como PNEA al primer trimestre de 2009 fue de 33 millones. De éstas, 5.7 
millones (17.3\% del total) fueron PNEA disponible y $82.7 \%$ PNEA no disponible, como muestra la gráfica 4 .

\section{Gráfica 4. Composición de la población no económicamente activa por no actividad, al primer trimestre de 2009.}

(Total: 33 millones de personas)

Disponible

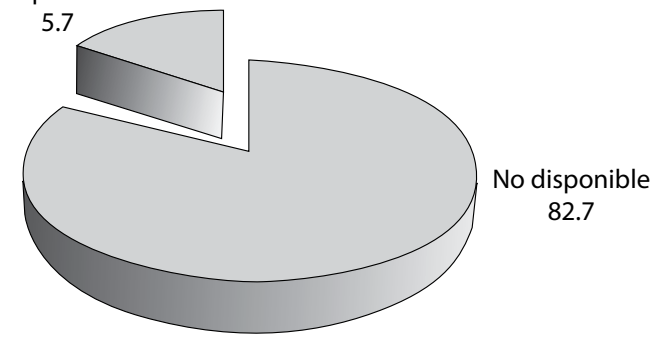

Fuente: STPS/INEGI (2009).

Es de sobra sabido que algunos países buscan reducir el nivel de sus tasas de desempleo, sobre todo cuando suben mucho, mediante múltiples subterfugios. Uno de ellos es precisamente trasladar parte de su PEA desempleada a PNEA (o PEI, como se le conoce internacionalmente), lo que provoca que se abulte el rubro de PNEA disponible. Se trata de personas que, aun reportando al encuestador que ya no buscan activamente trabajo, estarían dispuestas a tomarlo si se les ofreciese (Larraín y Sachs, 2002; Turnham, 1993). Así lo manifiesta, para el caso de América Latina y el Caribe durante la recesión de 2008-2009, el trabajo conjunto CEPAL/OIT (2009, p. 1).

El problema anterior se asocia de manera estrecha con el fenómeno de la histéresis, que caracteriza a los mercados laborales de todo el mundo y consiste en que, entre más tiempo pasa un individuo sin trabajar, menores son sus posibilidades de emplearse en el futuro, ya que por una parte pierde habilidades, lo que necesariamente lo desvincula del mercado y lo hace sentirse inseguro, y por la otra genera dudas entre sus potenciales empleadores (Blanchard y Summers, 1986). De ahí la importancia de reinsertar los trabajadores desalentados a la PEA mediante programas de capacitación, autoconfianza y búsqueda de trabajo, como consideran los seguros de desempleo. Negar la existencia de este estrato de desempleados sólo contribuye a la postergación de problemas incontenibles, como la pobreza extrema, la marginación social, la delincuencia y el hacinamiento carcelario. 
Si como medida de corrección estadística tanto los trabajadores ubicados por el INEGI en el renglón de PNEA disponible (desalentados) como los que llama trabajadores no remunerados (2.6 millones de personas en el primer trimestre de 2009, de los que la mayoría son trabajadores del campo que ayudan a su familia, al menos una hora en la semana de referencia, porque no tienen otra cosa que hacer) se reconocieran como población desocupada, el nivel de desocupación de México en el último trimestre de 2008 habría sido de 10.6 millones de personas (5.7 millones de PNEA disponible, 2.6 millones de trabajadores no remunerados y 2.3 millones de desocupados reconocidos por el INEGI).

Si la cifra anterior se divide entre la PEA ajustada de 50.9 millones (45.2 millones aceptados por la institución más 5.7 millones de PNEA disponible), se llega a una tasa de desocupación nacional de $20.8 \%$, en vez de la de $5.1 \%$, reportada por STPS/INEGI (2009) para el primer trimestre de 2009, como se muestra en el cuadro 4. Es decir, la tasa del INEGI podría estar subestimando la tasa real en una relación de 4 a 1. Esta realidad es independiente del hecho de que la tasa oficial es aceptada por organismos como la OIT. ${ }^{3}$ Aquí no se trata de lo que acepta la OIT como "razonable", ya que su metodología es excesivamente flexible, sino de lo que realmente muestra el mercado laboral.

\section{Cuadro 4. Recálculo de la tasa de desocupación de México}

al primer trimestre de 2009

(Millones de personas y porcentaje)

\begin{tabular}{|l|c|c|}
\hline \multicolumn{1}{|c|}{ Concepto } & INEGI & Recálculo \\
\hline 1. PEA & 45.2 & $50.9^{*}$ \\
\hline 2. Población desocupada & 2.3 & 10.6 \\
\hline a) Abierta & 2.3 & 2.3 \\
\hline b) PNEA disponible** & -- & 5.7 \\
\hline c) Trabajadores no remunerados & -- & 2.6 \\
\hline 3. Tasa de desempleo (2/1 en \%) & $\mathbf{5 . 1}$ & $\mathbf{2 0 . 8}$ \\
\hline
\end{tabular}

* PEA a partir de ENOE más PNEA disponible (trabajadores desalentados).

** Personas que no buscaron trabajo en el periodo de referencia inmediato, pero que aceptarían uno en cuanto se les ofreciera.

Fuente: a partir de STPS/INEGI (2009).

${ }^{3}$ Los cálculos de la elevada desocupación del país son también consistentes con el explosivo crecimiento del número de pobres en México durante 2006-2008 (unos 5 millones en valores absolutos), que llevó la tasa de pobreza alimentaria a aumentar de 13.8 a 18.2\%; la de pobreza de capacidades de 20.7 a $25.1 \%$ y la de pobreza de patrimonio de 42.6 a $47.4 \%$, según se desprende de la Encuesta Nacional de Ingresos y Gastos de los Hogares (INEGI, 2009b). 
Debe destacarse que las principales publicaciones metodológicas del INEGI señalan, convencidas, que la tasa de desocupación del país es baja no porque la metodología de levantamiento y el cuestionario de la ENOE estén mal, sino porque aun teniendo necesidad, mucha gente se autoexcluye del mercado laboral (PEA) con lo que automáticamente pasa a formar parte de la PNEA. Es decir, para el tamaño de la PEA de este país, según el INEGI "poca gente busca empleo" (INEGI, 2005c). Frente a estos argumentos, no existe poder humano capaz de hacer razonar a algunos técnicos y funcionarios de la institución sobre las debilidades de la metodología y las consecuencias de la inadecuada medición de las variables laborales. Todo lo contrario, la tasa de desocupación se utiliza oficialmente como un signo de fortaleza del mercado laboral mexicano, virtualmente sin parangón en el mundo.

Una discusión similar a la del inadecuado manejo de las estadísticas laborales y su pérdida de continuidad se presentó durante el cuarto trimestre de 2009, cuando un grupo de observadores y usuarios de información estadística manifestaron su preocupación respecto de la reducción en el número de variables que captará el Censo General de Población y Vivienda 2010. Sin inmutarse, las autoridades del instituto argumentaron que los propios académicos y usuarios ya habían aceptado que sólo las 27 preguntas fundamentales de demografía y vivienda se captaran a partir del censo (contra 52 en el básico del 2000), y las 75 restantes se obtuvieran a partir de una encuesta. La razón que se dio fue que el presupuesto para el levantamiento del censo, que aprobó la Cámara de Diputados en el marco del Presupuesto de Egresos de la Federación 2010, no había sido el solicitado por el INEGI, de 6 mil millones de pesos, sino "sólo de 4 mil 600 millones". Debe destacarse que, frente a esto, el instituto no hizo nada para modificar la decisión de los diputados, omitiendo el regateo, que es la forma en que se negocia el presupuesto en México. Es decir, pasa al país la factura de su desinterés por las estadísticas, ya que $72 \%$ de las variables que se capten en el censo de 2010 no son comparables con las de censos anteriores, en virtud de que provienen de una encuesta, además de que algunas son nuevas o diferentes a las anteriores. Entre éstas destacan las correspondientes al ingreso familiar, las laborales, las migratorias, las de desarrollo humano e incluso algunas sobre las características de las viviendas. Es como si se reeditara, en grande, la historia de la transición de la ENE a la ENOE.

\section{COMPARACIONES INTERNACIONALES}

En virtud de lo hasta ahora discutido respecto de las estadísticas laborales, las cifras de desocupación del país son muy poco comparables con las del resto de 
los miembros de la OCDE y el TLCAN. Por ejemplo, a pesar de ser la nación con menor desarrollo de la organización, México ostentaba la más baja tasa de desempleo (esa es la variable que usa la organización) hasta diciembre de 2009, junto con Luxemburgo e Islandia, como muestra la gráfica 5. A dicho problema de comparabilidad habría que sumar la virtual eliminación de la historia laboral de nuestras encuestas previas a 1995, mientras en los otros países se remontan hasta un siglo hacia atrás.

Es comprensible, por tanto, la insatisfacción de los expertos del Comité de Empleo, Trabajo y Asuntos Sociales de la OCDE (ELSA, por sus siglas en inglés) y del Bureau of Labor Statistics (BLS, 2009) de Estados Unidos, en cuyas series no pueden incluir a México, o lo hacen en sólo unos cuadros en virtud de que, a pesar de la importancia del país, su mercado laboral es poco comparable con el del resto del mundo, incluyendo los países con nivel similar de desarrollo. Además, aún seguimos discutiendo cómo medir la productividad laboral, a fin de distribuir sus beneficios, como muestra el segundo punto del Acuerdo Nacional Para la Productividad Laboral (STPS, 2009b), cuando para otros países esto se hace de manera periódica desde hace varias décadas (algo similar a lo que sucede con la medición del Índice Nacional de Precios al Consumidor).

Gráfica 5. Tasas de desempleo abierto en los países de la OCDE (2006) (Porcentaje respecto de la fuerza de trabajo civil: 15 a 64 años de edad)

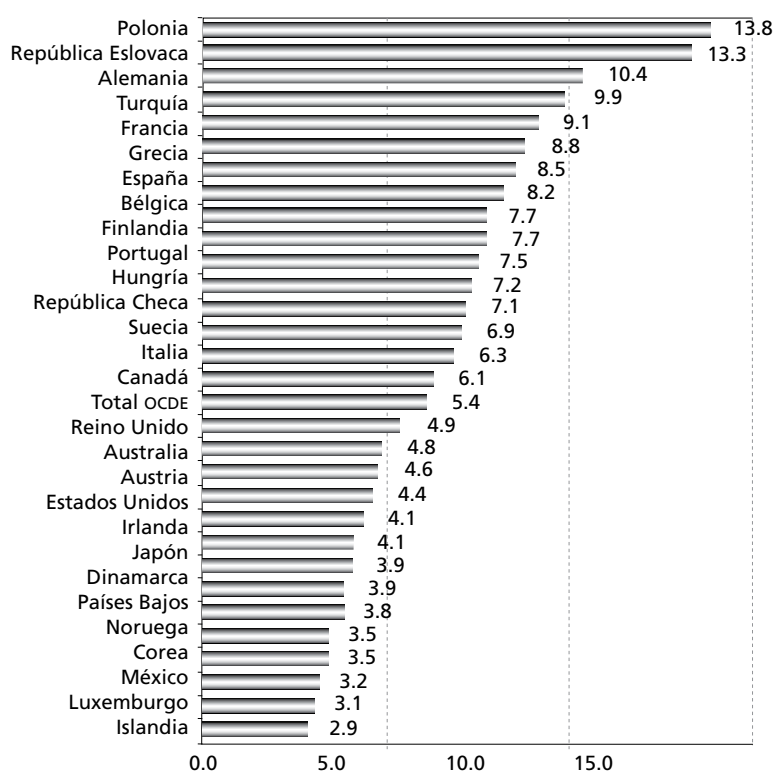

Fuente: OCDE (2009). 
En el desempleo de largo plazo (más de 12 meses) la paradoja se repite pero de manera más aguda. Como muestra la gráfica 6 , en dicha tasa México es un "privilegiado" de la OCDE, junto con Corea del Sur, ya que prácticamente no tienen ese tipo de desempleo. Empero, entre abril de 2008 y abril de 2009 el rubro de PNEA disponible (trabajadores supuestamente desalentados) pasó de 4.8 a 5.7 millones de personas, es decir creció 900 mil (STPS/INEGI, 2009).

Así como con las encuestas laborales en 2005, también hubo un cambio de series en el registro de trabajadores de empresas privadas más grande del país, el IMSS, en virtud de que en 2007 las autoridades de dicho instituto dieron a conocer que había problemas con la medición de los trabajadores urbanos eventuales, pues una gran parte de ellos eran en realidad urbanos permanentes.

\section{Gráfica 6. Desempleo de largo plazo en los países de la OCDE.}

(Personas desempleadas por 12 meses o más como

porcentaje del total de desempleados)

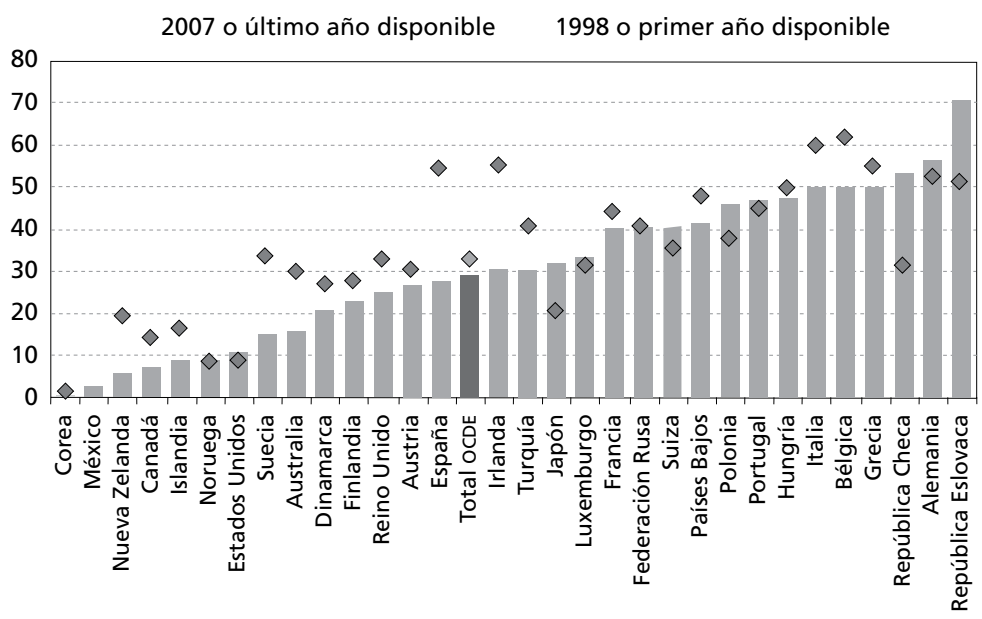

Fuente: OCDE (2009b).

Para corregir el error anterior, dijeron, se modificaría el método de cálculo, es decir, se dejaría de considerar el último reingreso del trabajador como cotizante al IMSS, y en su lugar se tomaría el último movimiento afiliatorio. Esto significó un aumento sustancial en el número de asegurados urbanos permanentes. Por ejemplo, al 15 de abril de 2008, la cifra ascendió a 842 mil, como muestra la gráfica 7 . 
Gráfica 7. Aumento en el número de cotizantes urbanos permanentes del IMSS debido a cambios en el método de contabilidad, 15 de abril de 2008
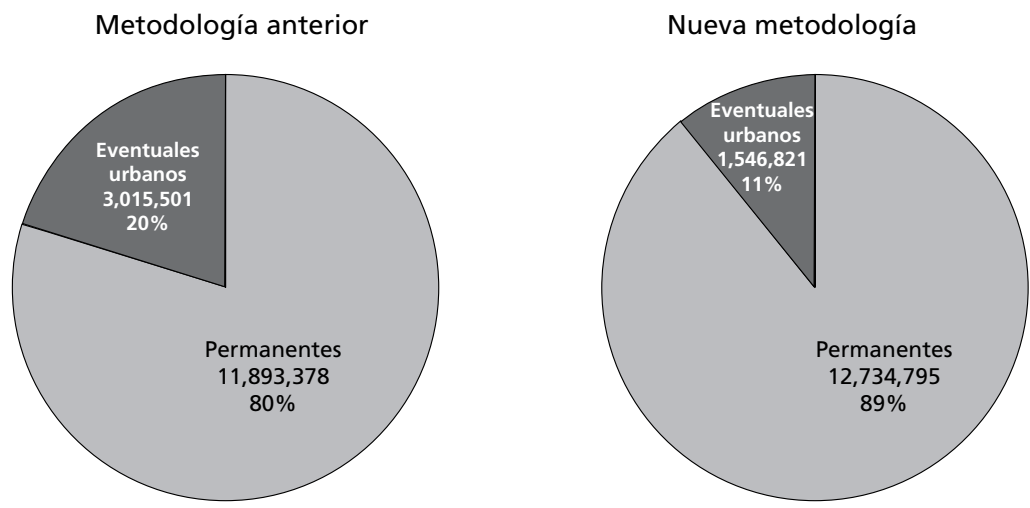

Fuente: IMSS (2008).

Casi como el INEGI, el IMSS reconstruyó sus series una década hacia atrás: a partir de julio de 1997 (IMSS, 2008). Con esto no sólo se pierde la información previa a dicho año, sino que se corrobora que lo que más interesa al gobierno federal es la imagen de creación neta de puestos de trabajo, sin reparar en que la calidad de éstos se deterioraría con el advenimiento de la siguiente recesión: entre julio de 2008 y mayo de 2009, mientras que de los 620 mil empleos perdidos por las empresas que cotizan al IMSS 536 mil fueron permanentes (91\% del total), sólo $51 \mathrm{mil}$ (9\% del total) fueron eventuales, como muestra la gráfica 8 .

Gráfica 8. Reducción del empleo formal privado registrado por el IMSS entre julio de 2008 y mayo de 2009

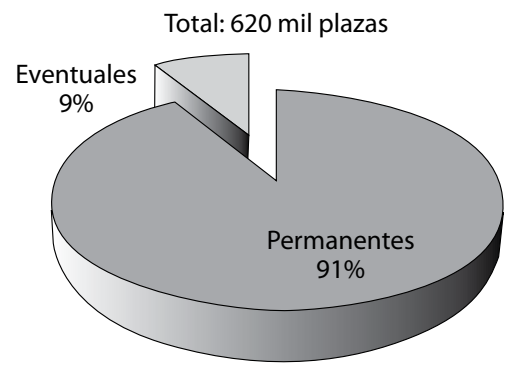

Fuente: STPS/IMSS (2009). 
Cuando se someten a prueba las variables del mercado laboral de México, a partir de sus relaciones con otras, y se le continúa comparando internacionalmente, se encuentran más inconsistencias. Por ejemplo, si se analiza la brecha ingresos-productividad laboral en los países de la OCDE, tomando como base de comparación a Estados Unidos, México aparece en último lugar, junto con Turquía y Polonia (gráfica 9). Esto sugiere que, entre menor es el nivel de desarrollo de un país, menos se refleja la productividad laboral en los ingresos de sus trabajadores. Asimismo, explica por qué la suma de sueldos, salarios y prestaciones sólo representa 32\% del PIB de México, pero llega a dos tercios en el caso de Estados Unidos, como muestran las cuentas nacionales de ambos países.

Al considerar que Polonia y Turquía ostentan las tasas más altas de desempleo de la OCDE, junto con República Eslovaca y Alemania, mientras México ostenta la tasa más baja, junto con Luxemburgo e Islandia (gráfica 5), la lógica deja de funcionar. Asimismo, surge la duda: ¿por qué la tasa de desempleo de México es menor a la de países como Dinamarca, Austria y Suecia, mientras su brecha ingresos-productividad es tres veces mayor? En términos generales, lo que refleja este análisis es que, mientras la tasa de desempleo, en la forma en que está calculada, ubica a México como si fuera un país con alto nivel de desarrollo, el pago a la productividad lo ubica como uno simplemente subdesarrollado.

Gráfica 9. Brecha porcentual ingresos-productividad de los países de la OCDE respecto al PNB per cápita de EUA en 2007

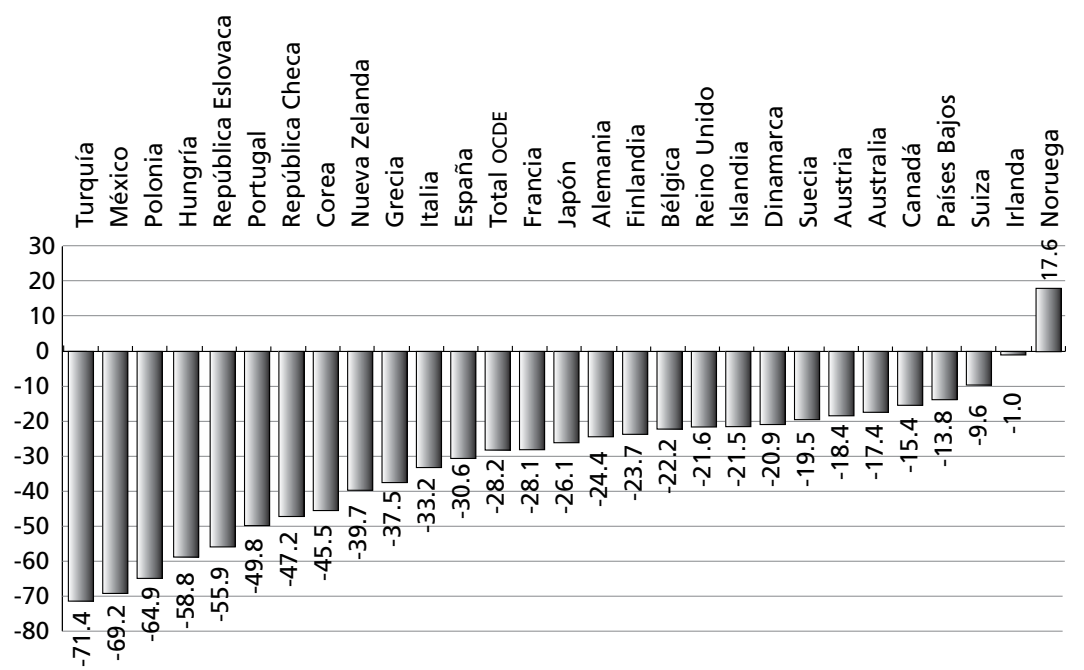

Fuente: OCDE (2009b). 
Otro caso es el de la tasa de crecimiento de largo plazo de un amplio grupo de economías de América Latina y el Caribe frente a su tasa de desempleo: mientras el nuestro es el que menos crece, su tasa de desempleo es la más baja; pero debería ser al contrario: en términos generales, las economías que más crecen son las que más empleos generan, como establecen lo mismo la teoría keynesiana (Keynes, 1936) que la neoclásica (Lerner, 1951; Phelps, 1972). O, como probó Okun (1962), a medida que la economía crece, la tasa de desempleo disminuye. Esto se presenta en la gráfica 10, cuyos resultados pueden leerse de la siguiente manera:

- La ecuación de la línea de tendencia, que es $Y=9.41-0.0307 X$, muestra que, aunque es muy baja la elasticidad entre crecimiento económico y reducción del desempleo, ésta existe: por cada punto porcentual que creció en promedio el PIB regional entre 2000 y 2008 , la tasa de desempleo urbano se redujo 0.0307. En otras palabras, aunque debe acelerarse mucho el crecimiento económico en la región para bajar apenas ligeramente la tasa de desempleo, prevalece la relación inversa entre ambas variables.

- Hay países outlier positivos (muy arriba y a la izquierda de la línea de tendencia), como Jamaica y Uruguay, que tienen tasas de crecimiento económico relativamente bajas (entre 1.4 y $3.1 \%$ ) asociadas con tasas de desempleo excesivamente altas (entre 12.2 y 13\%). Ente ellos, la variable aparentemente más incongruente es el crecimiento, el cual ayuda menos que en el promedio de la región a resolver los problemas del mercado laboral.

Gráfica 10. Relación entre las tasas de desempleo urbano y el crecimiento económico en países de América Latina y el Caribe

(Cifras promedio correspondientes al periodo 2000-2008)

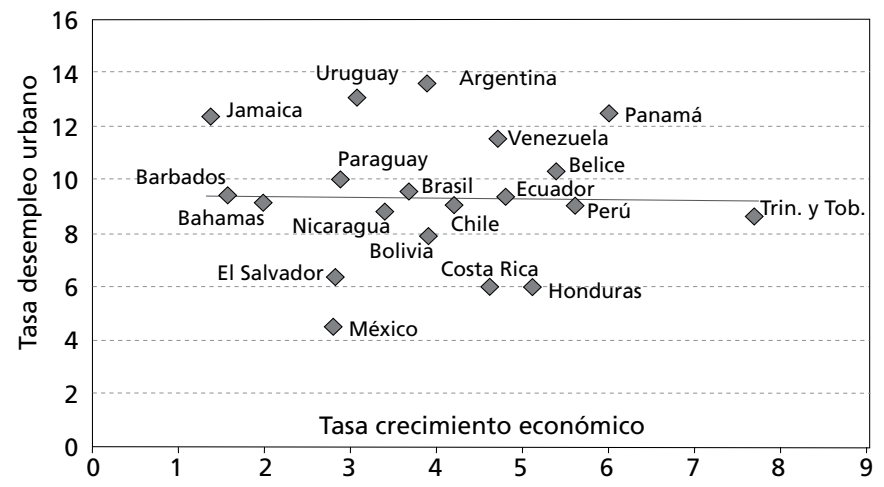

Fuente: a partir de CEPAL (2009). 
- Por el contrario, hay un outlier negativo (México), que con una de las tasas de crecimiento económico más bajas de la región (apenas 2.8\%) presenta también la tasa de desempleo urbano más baja (4.4\%). En este caso, la variable incongruente es la segunda. Así que el analista debe preguntarse dos cosas: ¿por qué el mercado laboral es tan benévolo con México, a pesar de que no ha hecho lo suficiente por crecer?, y ¿se estarán midiendo bien las tasas de desempleo o desocupación del país?

Finalmente, también sorprende que, mientras en los países de la OCDE, particularizando en la zona euro, con España como un miembro muy importante para México, y EUA (vecino y principal socio comercial del nuestro), la tasa armonizada de desempleo aumentó entre septiembre y diciembre de 2009 cuatro décimas de punto porcentual en promedio (cifras preliminares), proceso que estuvo asociado con los efectos rezagados de la crisis financiera estadounidense de 2008-2009 en el empleo, en México acusó una baja de siete décimas de punto porcentual de acuerdo con las cifras oficiales de cada país procesadas por la OCDE y presentadas en la gráfica 11 .

Gráfica 11. Variación de la tasa armonizada de desempleo en países de la OCDE de septiembre a diciembre de 2009

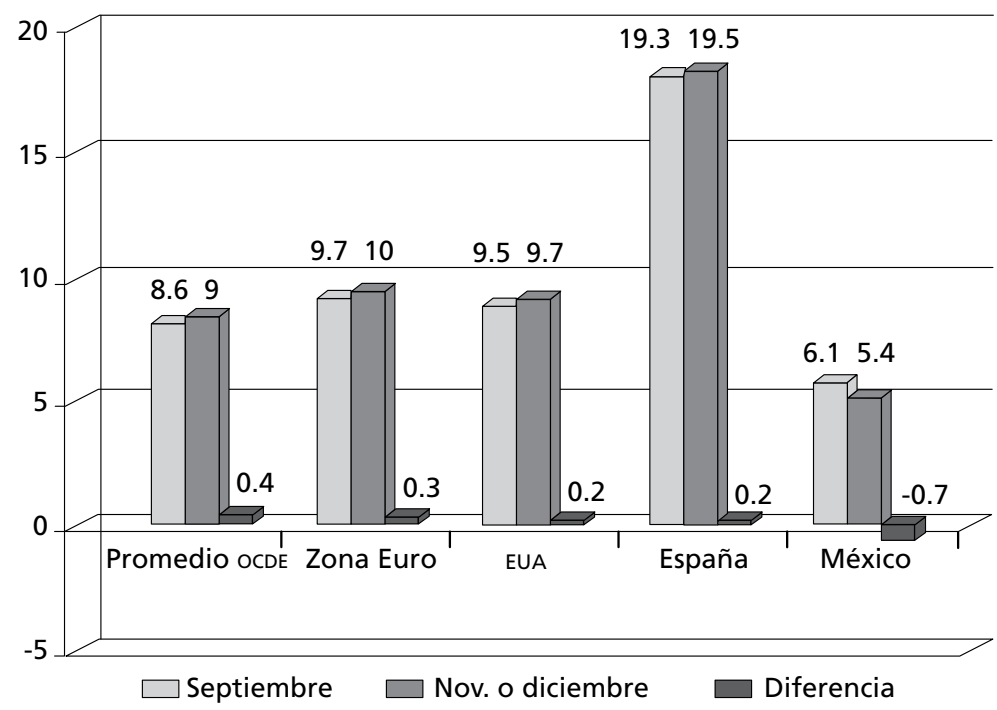

Fuente: OCDE (2010). 


\section{CONCLUSIONES}

Con su ingreso a la OCDE en 1994, así como con la puesta en operación del TLCAN en el mismo año, México eligió intensificar sus vínculos con las economías más desarrolladas del mundo y poner en operación una serie de políticas económicas y sociales que cumplieran con el artículo $1^{\circ}$. de la convención firmada en París, el 14 de diciembre de 1960, cuando se creó la OCDE. Dichas políticas se diseñaron para: $i$ ) lograr las más altas tasas de crecimiento económico y empleo sostenibles y un creciente estándar de vida, al tiempo que se mantiene la estabilidad económica y se contribuye al desarrollo de la economía mundial; ii) contribuir a la expansión de los países miembros y no miembros en el proceso de desarrollo económico, y iii) contribuir a la expansión del comercio mundial sobre bases multilaterales, no discriminatorias, de acuerdo con las obligaciones internacionales (OCDE, 1960).

Desafortunadamente, el proceso de actualización que impone a México su participación tanto en la OCDE como en el TLCAN incluye el mejoramiento de sus estadísticas a fin de que la contabilización de sus actividades económicas pueda someterse a comparaciones con sus principales socios y con el resto de países del mundo. No hay duda de que en los campos financiero y comercial se han logrado avances considerables en este terreno; pero en el social, la transición ha sido muy lenta, particularmente en lo que respecta a las variables del mercado laboral, donde resalta la imprecisión con que se mide la tasa de desocupación/desempleo.

Dicha imprecisión obedece a que México, aunque parte de los criterios básicos de la OIT para considerar a una persona desempleada -se encuentra en edad de trabajar, tiene plena disponibilidad para laborar y busca activamente emplearse-, pone en práctica excepciones importantes al concepto que lo acercan más a los países con menor desarrollo del mundo que a los miembros de la OCDE y el TLCAN. Es decir, deja de hablar de desempleo y lo sustituye por desocupación; fija la edad mínima para trabajar en 14 y no en 15 años, como sugieren las estadísticas de la OCDE; no pone límite a la edad máxima para mantenerse en el mercado laboral, que en las estadísticas de la OCDE es de 64 años, y fija el tiempo mínimo de trabajo semanal necesario para no considerar desocupada a una persona en apenas una hora, no obstante que en las estadísticas de la OCDE es de 15, y en particular en Estados Unidos de 16. Éste es evidentemente el punto más controversial de la medición del desempleo.

Adicionalmente, recurre de manera extensiva al subterfugio estadístico de trasladar personas en edad de trabajar de la PEA a la PNEA, ya sea disponible o 
no disponible, en cuanto declaran al entrevistador del INEGI que han dejado de realizan acciones para encontrar un empleo; clasifica a las personas que realizan alguna labor familiar no remunerada o de ayuda a amigos sin recibir pago por al menos una hora en la semana de referencia como ocupada, y se refiere al subempleo como el de personas que, estando empleadas, buscan una segunda ocupación, mientras en la OCDE dicho concepto hace alusión a quienes trabajan menos de 30 horas a la semana.

Si los errores anteriores se corrigieran, la PEA del país sería de más de 50 millones de personas en el primer trimestre de 2009 y no de 45.3 millones, como contabiliza el INEGI mediante la ENOE. Asimismo, la tasa de desocupación superaría $20 \%$ y la de subocupación sería aún mayor. Efectivamente, estos niveles, que parecen escandalosos, tendrían la virtud de ser consistentes con los que arroja la metodología de la OCDE, cuyos países miembros, con excepción de México, comprueban en gran medida los resultados de sus encuestas de empleo y desempleo con el número y ritmo de quienes solicitan el seguro de desempleo, otro gran pendiente social de México.

Por otra parte, es claro que no sólo no conviene a los trabajadores e investigadores del sector laboral el status quo en materia de estadísticas laborales, sino al país en su conjunto, ya que las cifras sobre el número de personas desempleadas o desocupadas, al subestimar la realidad, no permiten planear ni tomar medidas de política económica y laboral correctivas.

Es tiempo de que los estadígrafos y tomadores de decisiones del INEGI recapaciten sobre la invalidez de los argumentos que dan a los expertos nacionales e internacionales que se muestran inconformes con la medición de la tasa de desocupación de la ENOE. Éstos consisten en que dicha medición cumple con las normas establecidas por la OIT; toma en cuenta diversos elementos en la materia de la OCDE y el TLCAN y, si es baja, se debe a que los encuestados, aun estando en edad y condiciones de trabajar, responden al encuestador del INEGI que han decidido dejar de buscar empleo o generarse una autoocupación.

La antepropuesta de reforma laboral enviada al Congreso en febrero de 2009 es una excelente oportunidad para crear un parteaguas en este campo y para que la transformación del INEGI en Instituto Nacional de Estadística y Geografía llevada a cabo en 2008 lo lleve a ser la autoridad estadística independiente que la sociedad espera. Por supuesto, éste es un caso más de coordinación entre diferentes esferas de la sociedad -técnicos gubernamentales, académicos, empresarios, trabajadores-, pero que para ponerse en marcha requiere de una voluntad política. 
70 ECONOMÍA: TEORÍA Y PRÁCTICA • Nueva Época, número 31, julio-diciembre 2009

\section{REFERENCIAS BIBLIOGRÁFICAS}

Blanchard, O., y L. Summers (1986), "Hysteresis and the European Unemployment Problem", NBER Macroeconomics Annual, Cambridge, MIT Press.

BLS (Bureau of Labour Statistics) (2009), www.bls.gov (consultado en abril).

CEPAL (Comisión Económica para América Latina y el Caribe) (2009), Estudio económico de América Latina y el Caribe 2008-2009, Santiago de Chile, julio.

— IOIT (2009), "Coyuntura laboral en América Latina y el Caribe", Boletín CEPAL/ OIT, Núm. 2, septiembre.

CESOP (Centro de Estudios Sociales y de Opinión Pública) (2009), Reforma laboral. Carpeta Informativa, México, febrero.

Conapo (Consejo Nacional de Población) (2009), proyecciones de la PEA 2005-2050, www.conapo.gob.mx (consultado en junio).

Diario Oficial de la Federación (2008), "Ley del Sistema Nacional de Información Estadística y Geográfica", México, 16 de abril.

Gutiérrez R., Roberto (2009), "La reforma petrolera de México. ¿Dos sexenios sin política energética?, Argumentos, Núm. 58, septiembre-diciembre.

IMSS (2008), "Revisión de la metodología para la obtención de la cifra de trabajadores permanentes y eventuales urbanos (TPEU) asegurados del IMSS", presentación, noviembre.

- (2009), www.imss.gob.mx (consultado en abril).

INEGI (1998), Indicadores Económicos de Coyuntura por Entidad Federativa, México, enero.

- (2005 a), Metodología de la ENOE, www.inegi.org.mx (consultado en mayo).

— (2005b), "Cuestionario de Ocupación y Empleo (básico)”, www.inegi.org.mx (consultado en mayo).

- (2005 c), Encuesta Nacional de Ocupación y Empleo 2005. Una nueva encuesta para México, México.

- (2009), Encuesta Nacional sobre Ocupación y Empleo (ENOE), cifras al primer trimestre de 2009, México, www.stps.gob.mx (consultado en julio).

- (2009 a), Anuario Estadístico de los Estados Unidos Mexicanos 2008, México. (2009 b), Encuesta Nacional de Ingresos y Gastos de los Hogares 2008, México. (2009 c), "Indicadores oportunos sobre ocupación y empleo. Cifras preliminares durante julio de 2009", www.inegi.org.mx, comunicado Núm. 239/09, 26 de agosto (consultado en agosto).

INEGI (2009d), "Indicadores oportunos sobre ocupación y empleo. Cifras preliminares durante septiembre de 2009”, www.inegi.org.mx, comunicado Núm. 274/09, 21 de octubre (consultado en octubre). 
INEGI / STPS (2009b) “Estadísticas”, www.inegi.org.mx (consultado en mayo). / IMSS (2009), "Estadísticas”, www.stps.gob.mx (consultado en junio).

ITU (International Telecommunications Union) (2009), Measuring the Information Society - The ICT Development Index, Ginebra.

Keynes, John M. (1936), The General Theory of Employment, Interest and Money, Nueva York, Harcout Brace.

Larraín, Felipe, y Jeffrey Sachs (2002), Macroeconomía en la economía global, México, Prentice Hall.

Lerner, Abba P. (1951), Economics of Employment, McGraw-Hill.

Lozano Alarcón, Javier (2009), Hacia una reforma laboral para la productividad y la prevision social, México, STPS, 9 de febrero.

Martin, John (1994), “The Extent of High Unemployment in OECD Countries”, Reducing Unemployment: Current Issues and Policy Options, Reserva Federal de Kansas. OCDE (1960), "Convention of the STPS”, París, diciembre.

(2009 a), OECD Economic Outlook, París, junio.

(2009b), www.oecd.org (consultado en junio).

(2010), Labour Force Statistics, www.oecd.org (consultado en enero).

OIT (1986), "Fuentes y métodos estadísticos", Encuesta de Hogares, Ginebra, Vol. 3.

(1996), "Sources and Methods", Labor Statistics, Ginebra, Vol. 5.

Okun, A. (1962), "Potential GNP: its Measurement and Significance", Proceedings of the Business and Economic Statistics, American Statistical Association, Alejandría.

Pemex (Petróleos Mexicanos) (2009), Dictámenes de la reforma energética, www.pemex. com.mx (consultado en julio).

Phelps, Edmund S. (1972), Inflation Policy and Unemployment Theory. The Cost-Benefit Approach to Monitory Planning, Londres, Macmillan.

Portes, A. (1994), "When more can be less: labor standards, development, and the informal economy", en Rakowski, C. A. (editor), Contrapunto: The informal sector debate in Latin America, Albany, Nueva York, State University of New York Press.

Presidencia de la República (2009), Programas sectoriales www.presidencia.gob.mx/ progsectoriales (consultado en julio).

STPS (1994), El mercado de trabajo en México (1970-1992), México.

- (2009 a), Estadísticas, www.stps.gob.mx (consultado en junio).

(2009b), www.empleo.gob.mx (consultado en mayo).

- (2009 c), Acuerdo Nacional para la Productividad Laboral, México, mayo.

(2009 d), "Comentarios de la STPS sobre la tasa de desocupación del mes de julio”, www.stps.gob.mx, Boletín 109, 26 de agosto. 
72 ECONOMÍA: TEORÍA Y PRÁCTICA • Nueva Época, número 31, julio-diciembre 2009

Turnham, D. (1993), Employment and Development. A New Review of Evidence, París, OCDE.

Vela, Fortino (2008), "Cambio en la estructura por edad y su efecto en el desempleo de los jóvenes urbanos", en Vela Fortino, (coord.), La dinámica demográfica y su impacto en el mercado laboral de los jóvenes, México, UAM-Xochimilco. 